\title{
Super-Resolution and Joint Segmentation in Bayesian Framework
}

\author{
Fabrice Humblot ${ }^{* \dagger}$ and Ali Mohammad-Djafari* \\ ${ }^{*}$ LSS / UMR 8506 (CNRS-Supélec-UPS), \\ 91192 Gif-sur-Yvette Cedex, France. \\ ${ }^{\dagger} D G A / D E T / S C E T / C E P / A S C / G I P$, \\ 94114 Arcueil, France.
}

\begin{abstract}
.
This communication presents an extension to a super-resolution (SR) method we previously exposed in [1]. SR techniques involve several low-resolution (LR) images in the reconstruction's process of a high-resolution (HR) image. The LR images are assumed to be obtained from the HR image through optical and sensor blurs, shift movement and decimation operators, and finally corruption by a random noise. Moreover, the HR image is assumed to be composed of a finite number of homogeneous regions. Thus, we associate to each pixel of the HR image a classification variable which is modeled by a Potts Markov field. The SR problem is then expressed as a Bayesian joint estimation of the HR image pixel values, its classification labels variable, and the problem's hyperparameters. These estimations are performed using an appropriate algorithm based on hybrid Markov Chain Monte-Carlo (MCMC) Gibbs sampling.

In this study, we distinguish two kinds of region's homogeneity: the first one follows a constant model, and the second a bilinear model. Our previous work [1] only deals with constant model. Finally we conclude this work showing simulation results obtained with synthetic and real data.
\end{abstract}

Keywords: Super-resolution, MCMC Gibbs sampling, joint estimation, classification and segmentation.

PACS: $87.57 . \mathrm{Gg}$

\section{INTRODUCTION}

This communication deals with super-resolution (SR) reconstruction of an image from several low-resolution (LR) images. To obtain an image well reconstructed by a SR process, one needs first to register LR images, and thus, to know the transformations that permit to position all observed images on a common grid of reference. To perform this, we used the phase correlation principle, a frequency domain method that estimates a global shift between two images. We do not discuss this aspect here. The reader can refer to [2] for more information about it.

The first approach related to multi-frame SR restoration computes a solution in frequency-domain [3]. However, more recent works have found the spatial-domain methods more promising, in particular the Bayesian framework. For a recent and detailed overview of the state of the art, we suggest [4].

Spatial-domain SR restoration techniques are based on an observation model that links LR images and HR image via optical and sensor blurs, shift movement and decimation operators. This is also the model we choose. To our knowledge, a lot of well known and popular existing HR reconstruction methods are often based on a regularized 
approach [5, 6, 7]. In this work, which is an extension of our previous work [1], we use a Bayesian estimation framework which gives the possibility to account for a broad range of prior models to formulate inverse problem of multi-frame SR restoration (we can mention for instance the previous works $[8,9]$ ). The innovation of the present study lies in the definition of region's homogeneity which follows a bilinear model here, instead of a constant one in [1].

Therefore, the prior distribution of the pixels is modeled by a Finite Mixture Model (FMM) so, a finite number of classes is used for their classification. The Bayesian framework with the FMM is a common tool for classification but usually, the discrete variable associated to each pixel is supposed to be i.i.d. [8]. Our Potts Markov Model (PMM) for these variables allows to consider the spatial correlation of the pixels.

Then, the prior information we put on the distribution of the pixels of the HR image brings about a hierarchical Markov model. This model allows to estimate in an iterative way the HR image, the classification labels, and the parameters of the noise and prior models. This method gives an absolutely unsupervised HR image estimation and segmentation. The hierarchical structure of the model can be suitably carried out using the Markov Chain Monte-Carlo (MCMC) Gibbs sampling.

The main original contribution of this communication, compared to the work presented by the authors in [1], is the introduction of a more general and more appropriate prior model. However, to present this new contribution as a self-containing paper, we give here the main steps of the modeling and the Bayesian framework which are the same as in paper [1], and which we suggest to readers for the details. Finally, in this work, we give a comparison of results obtained with this new model and with the previous one on simulated and real data.

\section{FORWARD MODEL AND BAYESIAN FRAMEWORK}

The SR reconstruction of an image is an inverse problem. Its joint forward model can be formulated using the following model that links the LR images and the HR image:

$$
\boldsymbol{g}_{i}=\boldsymbol{D} \boldsymbol{M}_{i} \boldsymbol{B} \boldsymbol{f}+\boldsymbol{\epsilon}_{i}=\boldsymbol{H}_{i} \boldsymbol{f}+\boldsymbol{\epsilon}_{i}, \quad i=1, \cdots, M .
$$

In this equation $\boldsymbol{f}$ is the HR image, $\left\{\boldsymbol{g}_{i, i=1, \ldots, M}\right\}$ are the $M$ LR images, $\boldsymbol{B}$ stands for the optical blurs (low pass filtering operator) as well as the integration on the LR pixel detector surface, $\boldsymbol{M}_{i}$ are shift movement operators, $\boldsymbol{D}$ is a decimation operator, $\boldsymbol{\epsilon}_{i}$ are the additive noises representing all the modeling errors, and finally, $\boldsymbol{H}_{i}=\boldsymbol{D} \boldsymbol{M}_{i} \boldsymbol{B}$ represents the compound operator connecting the HR image $\boldsymbol{f}$ to one LR image $\boldsymbol{g}_{i}$.

Moreover, we also use the following combination:

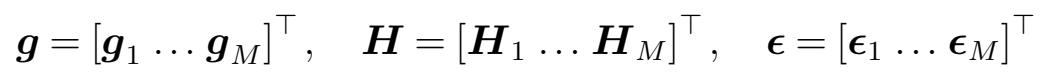

to rewrite the equation (1) as: $\boldsymbol{g}=\boldsymbol{H} \boldsymbol{f}+\boldsymbol{\epsilon}$. We use the forward model when we compute $\boldsymbol{g}$ knowing $\boldsymbol{f}$ and $\boldsymbol{H}$, and we resolve the analogous inverse problem of multi-frame SR restoration when we estimate $\boldsymbol{f}$ given $\boldsymbol{H}$ and $\boldsymbol{g}$.

As an illustration, the figures on the right side of the following page show a HR image $\boldsymbol{f}$, and one of the corresponding LR images $\boldsymbol{g}_{i}$ for a decimation factor equals to 5 . Both 
images are shown at the same size to emphasize the difference of resolution.

The Bayesian estimation framework for SR reconstruction can be summarized as follows:

- Use the forward model (1) and some assumptions on the noise to obtain the likelihood $p\left(\boldsymbol{g} \mid \boldsymbol{f}, \boldsymbol{\theta}_{\epsilon}\right)$ where $\boldsymbol{\theta}_{\epsilon}$ represents the parameters of the probability distribution of the noise.

- Use all the prior information or the desired properties for the solution to assign an a priori probability distribution $p\left(\boldsymbol{f} \mid \boldsymbol{\theta}_{f}\right)$ where $\boldsymbol{\theta}_{f}$ is its parameters.

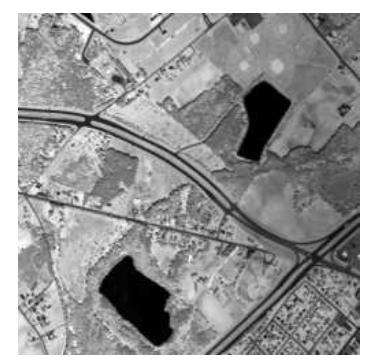

$\boldsymbol{f}, 250 \times 250$ pixels

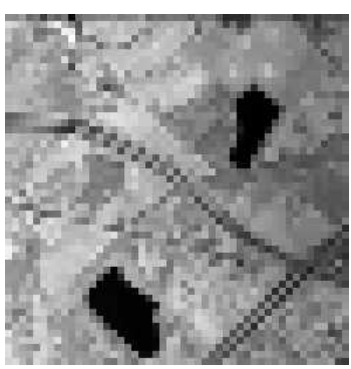

$\boldsymbol{g}_{1}, 50 \times 50$ pixels

\section{A PRIORI MODEL OF THE HR IMAGE}

As in [1], we suppose in our model that the image $f(\boldsymbol{r}), \boldsymbol{r} \in \mathcal{R}$, is made-up of a finite set $K$ of homogeneous regions $\mathcal{R}_{k}$ with given labels $z(\boldsymbol{r})=k, \quad k=1, \cdots, K$. We note $\mathcal{R}_{k}=\{\boldsymbol{r}: z(\boldsymbol{r})=k\}$ and assume $\mathcal{R}=\cup_{k=1}^{K} \mathcal{R}_{k}$ and the pixel values corresponding to a region $k: \boldsymbol{f}_{k}=\left\{f(\boldsymbol{r}): \boldsymbol{r} \in \mathcal{R}_{k}\right\}$ with $\boldsymbol{f}=\cup_{k=1}^{K} \boldsymbol{f}_{k}$. Region's homogeneity is defined in section 4.

Such images can be properly model in an effective and general way using the Hidden Markov Modeling (HMM). The central concept is to suppose that all the pixel values $\boldsymbol{f}_{k}$ of an homogeneous region $k$ follow a given probability law, that we chose to be a Gaussian $\mathcal{N}\left(m_{k}, \boldsymbol{\Sigma}_{k}\right)$ where $m_{k}$ is a vector of size $n_{k}=\left|\mathcal{R}_{k}\right|$ which is the number of pixels in region $k$, with $\sum_{k=1}^{K} n_{k}=|\mathcal{R}|=n$, the total number of pixels of the HR image $\boldsymbol{f}$, and $\boldsymbol{\Sigma}_{k}$ is assumed either diagonal $\boldsymbol{\Sigma}_{k}=\sigma_{k}^{2} \boldsymbol{I}$, or representing the covariance matrix of a MRF parameterized by $\left\{\sigma_{k}, \rho_{k}\right\}$. More specifically:

$$
p\left(\boldsymbol{f}_{k}\right) \propto \exp \left[\frac{-1}{2 \sigma_{k}^{2}}\left(\sum_{\boldsymbol{r} \in \mathcal{R}_{k}}\left(f(\boldsymbol{r})-m_{k}(\boldsymbol{r})\right)-\rho_{k} \beta \boldsymbol{r} \sum_{\boldsymbol{s} \in \mathcal{V}(\boldsymbol{r})}\left(f(\boldsymbol{s})-m_{k}(\boldsymbol{s})\right)\right)^{2}\right]
$$

where in the following we consider two cases for $m_{k}(\boldsymbol{r})$ which are detailed in section $\mathbf{4}$, $\mathcal{V}(\boldsymbol{r})$ represents neighboring pixels of $\boldsymbol{r}$ with $|\mathcal{V}(\boldsymbol{r})|=4$, and where $\beta \boldsymbol{r}$ is a coefficient depending on pixel $\boldsymbol{r}$. Let $n_{\boldsymbol{r}}$ be $\operatorname{Card}\left(\mathcal{V}(\boldsymbol{r}) \cap \mathcal{R}_{k}\right)$, which is the number of neighboring 
pixels of the pixel $\boldsymbol{r}$ that belong to the same region $\mathcal{R}_{k}$. $\beta \boldsymbol{r}$ equals to $\frac{1}{n_{\boldsymbol{r}}}$ if $n_{\boldsymbol{r}} \neq 0$ and equals to 0 otherwise.

Moreover, we assume that the pixels in different regions are independent:

$$
p(\boldsymbol{f} \mid \boldsymbol{z})=\prod_{k=1}^{K} p\left(\boldsymbol{f}_{k}\right)=\prod_{k=1}^{K} \mathcal{N}\left(\boldsymbol{f}_{k} \mid m_{k} \mathbf{1}, \boldsymbol{\Sigma}_{k}\right)
$$

where $\Sigma_{k}$ is a covariance matrix that is fitting, and which depends on the model of dependency that has been chosen. In the simulations of section $\mathbf{5}$, we used a first order Markov model limited to the four nearest neighbors.

Note that $p(f(\boldsymbol{r}) \mid z(\boldsymbol{r})=k)=\mathcal{N}\left(\boldsymbol{f}_{k}(\boldsymbol{r}) \mid m_{k}, \sigma_{k}^{2}\right)$ which results to the following FMM:

$$
p(f(\boldsymbol{r}))=\sum_{k=1}^{K} p(z(\boldsymbol{r})=k) \mathcal{N}\left(f_{k}(\boldsymbol{r}) \mid m_{k}, \sigma_{k}^{2}\right) .
$$

Then, we require to model the probability distribution $p(Z(\boldsymbol{r})=z(\boldsymbol{r}), \boldsymbol{r} \in \mathcal{R})$ of the random vector $\boldsymbol{Z}=\{Z(\boldsymbol{r}): \boldsymbol{r} \in \mathcal{R}\}$ which we note now $p(\boldsymbol{z})$. Again, as in [1], the main difference with the classical FMM is that here, we model the structural dependency of $\{Z(\boldsymbol{r}), \boldsymbol{r} \in \mathcal{R}\}$ by the following PMRF:

$$
p(\boldsymbol{z}) \propto \exp \left[\alpha \sum_{\boldsymbol{r} \in \mathcal{R}} \sum_{\boldsymbol{s} \in \mathcal{V}(\boldsymbol{r})} \delta(z(\boldsymbol{r})-z(\boldsymbol{s}))\right] .
$$

The first order Markov model, which supposes a 4-connexity, assumes that each label value $z(\boldsymbol{r})$ of a pixel at position $\boldsymbol{r}$ is function of the values of its four closest pixels $z(\boldsymbol{s})$, $s \in \mathcal{V}(\boldsymbol{r})$. The parameter $\alpha$ controls the mean value of the classes' sizes. Thus, increasing $\alpha$ makes the different classes become more homogeneous (in practice we choose $\alpha=2$ ). The Hammerslay-Clifford equivalence of the Gibbs and Markov random fields allows to write:

$$
p(z(\boldsymbol{r}) \mid z(\boldsymbol{s}), \boldsymbol{s} \in \mathcal{R}) \propto \exp \left[\alpha \sum_{\boldsymbol{s} \in \mathcal{V}(\boldsymbol{r})} \delta(z(\boldsymbol{r})-z(\boldsymbol{s}))\right]
$$

which shows that the probability of obtaining a label $z(\boldsymbol{r})$ for a pixel is related to the number of neighboring pixels having the same label. The Kronecker delta $\delta($.$) is defined$ by $\delta(0)=1$, and $\delta(t)=0$ for $t \neq 0$.

The last part of our a priori modeling is to assign a prior probability law $p(\boldsymbol{\theta})$ to the hyperparameters $\boldsymbol{\theta}$. We choose here to use the conjugate priors for simplicity [10]. Then, we need to assign prior probability laws to the means $m_{k}$, to the covariance matrices $\Sigma_{k}$, and also to the covariance matrices of the noises $\Sigma_{\epsilon i}$. The conjugate priors for the means $m_{k}$ are commonly the Gaussians $\mathcal{N}\left(m_{k} \mid m_{k 0}, \sigma_{k 0}^{2}\right)$ and those for the covariance matrices $\boldsymbol{\Sigma}_{k}$ are the inverse Wishart's $\mathcal{I} \mathcal{W}\left(\Sigma_{k} \mid \alpha_{k 0}, \boldsymbol{\Lambda}_{k 0}\right)$. We assume that the noises $\boldsymbol{\epsilon}_{i}$ are independent, centered and Gaussian with covariance matrices $\boldsymbol{\Sigma}_{\epsilon i}$ which, hereafter, are also assumed to be diagonal giving $\boldsymbol{\Sigma}_{\epsilon i}=\sigma_{\epsilon i}^{2} \boldsymbol{I}$. We note $\boldsymbol{\theta}_{\epsilon}=\left\{\sigma_{\epsilon i}^{2}, i=1, \cdots, M\right\}$. Thus, the conjugate priors of noise variances $\sigma_{\epsilon i}^{2}$ are the inverse Gamma laws $\mathcal{I} \mathcal{G}\left(\sigma_{\epsilon_{i}}^{2} \mid \alpha_{0}^{\epsilon_{i}}, \beta_{0}^{\epsilon_{i}}\right)$. See [1] for the detailed expressions of these probability density functions. 


\section{POSTERIOR PROBABILITY AND ESTIMATION ALGORITHM}

The likelihood is obtained through the observation model (1). Assuming the noise Gaussian, we have:

$$
p\left(\boldsymbol{g} \mid \boldsymbol{f}, \boldsymbol{\theta}_{\epsilon}\right)=\prod_{i=1}^{M} p\left(\boldsymbol{g}_{i} \mid \boldsymbol{f}, \boldsymbol{\Sigma}_{\epsilon i}\right)=\prod_{i=1}^{M} \mathcal{N}\left(\boldsymbol{g}_{i} \mid \boldsymbol{H}_{i} \boldsymbol{f}, \sigma_{\epsilon}^{2}\right) \text {. }
$$

Then, the equations (2) and (3) give $p(\boldsymbol{f} \mid \boldsymbol{\theta})$. Thus, the hyperparameters become $\boldsymbol{\theta}_{f}=\left\{\left(m_{k}, \sigma_{k}^{2}, \rho_{k}\right), k=1, \cdots, K\right\}$.

Next, we have the equation (4) that gives $p(\boldsymbol{z})$ and which is a result of the PMRF model we put on labels. Also, we defined the conjugate prior laws for the hyperparameters at the end of section 3. Finally, we obtain the following joint posterior law of $\boldsymbol{f}, \boldsymbol{z}$ and $\boldsymbol{\theta}=\left(\boldsymbol{\theta}_{\epsilon}, \boldsymbol{\theta}_{f}\right): \quad p(\boldsymbol{f}, \boldsymbol{z}, \boldsymbol{\theta} \mid \boldsymbol{g}) \propto p\left(\boldsymbol{g} \mid \boldsymbol{f}, \boldsymbol{\theta}_{\epsilon}\right) p\left(\boldsymbol{f} \mid \boldsymbol{z}, \boldsymbol{\theta}_{f}\right) p(\boldsymbol{z}) p(\boldsymbol{\theta})$.

This posterior law (6) is used through an hybrid Markov Chain Monte-Carlo (MCMC) Gibbs sampling to generate iteratively samples $(\boldsymbol{f}, \boldsymbol{z}, \boldsymbol{\theta})$ from which we can define the estimates $(\widehat{\boldsymbol{f}}, \widehat{\boldsymbol{z}}, \widehat{\boldsymbol{\theta}})$. We call it MAP-Gibbs sampling:

$$
\left\{\begin{array}{ccc}
\boldsymbol{f} & =\arg \max _{\boldsymbol{f}}\{p(\boldsymbol{f} \mid \boldsymbol{z}, \boldsymbol{\theta}, \boldsymbol{g})\} \\
\text { sample } \boldsymbol{\theta} & \text { using } p(\boldsymbol{\theta} \mid \boldsymbol{f}, \boldsymbol{z}, \boldsymbol{g}) \\
\text { sample } & \boldsymbol{z} & \text { using } p(\boldsymbol{z} \mid \boldsymbol{f}, \boldsymbol{\theta}, \boldsymbol{g}) .
\end{array}\right.
$$

This algorithm allows to estimate the HR image $f(\boldsymbol{r})$, the structure of each homogeneous region modeled through $z(\boldsymbol{r})$, and estimate also the corresponding hyperparameters $m_{k}$, $\sigma_{k}^{2}, \rho_{k}$ and $\sigma_{\epsilon i}^{2}$ through an unsupervised iterative process.

Detailed expressions of the posteriors $p(\boldsymbol{\theta} \mid \boldsymbol{f}, \boldsymbol{z}, \boldsymbol{g}), p(\boldsymbol{z} \mid \boldsymbol{f}, \boldsymbol{\theta}, \boldsymbol{g})$ and the sampling algorithms are given in [1]. Here, we only detail the evaluation of $\widehat{\boldsymbol{f}}$ when $\boldsymbol{\theta}$ and $\boldsymbol{z}$ are known:

$$
\widehat{\boldsymbol{f}}=\arg \max _{\boldsymbol{f}}\{p(\boldsymbol{f} \mid \boldsymbol{g}, \boldsymbol{z}, \boldsymbol{\theta})\}=\arg \min _{\boldsymbol{f}}\{\boldsymbol{J}(\boldsymbol{f} \mid \boldsymbol{g}, \boldsymbol{z}, \boldsymbol{\theta})\} .
$$

It can be shown that:

$$
\begin{aligned}
\boldsymbol{J}(\boldsymbol{f} \mid \boldsymbol{g}, \boldsymbol{z}, \boldsymbol{\theta}) & =\|\boldsymbol{g}-\boldsymbol{H} \boldsymbol{f}\|_{\boldsymbol{\Sigma}_{\boldsymbol{\epsilon}}}^{2}+\|\boldsymbol{f}-\boldsymbol{m}\|_{\boldsymbol{\Sigma}_{\boldsymbol{f}}}^{2} \\
& =\sum_{i=1}^{M} \frac{1}{\sigma_{\epsilon i}^{2}}\left\|\boldsymbol{g}_{i}-\boldsymbol{H}_{i} \boldsymbol{f}\right\|^{2}+\sum_{k=1}^{K} \frac{1}{\sigma_{k}^{2}} \sum_{\boldsymbol{r} \in \mathcal{R}_{k}}\left(\tilde{f}(\boldsymbol{r})-\rho_{k} \beta \boldsymbol{r}\left(\sum_{\boldsymbol{s} \in\left(\mathcal{V}(\boldsymbol{r}) \cap \mathcal{R}_{k}\right)} \tilde{f}(\boldsymbol{s})\right)(\boldsymbol{p})\right.
\end{aligned}
$$

where $\tilde{f}(\boldsymbol{r})=f(\boldsymbol{r})-m(\boldsymbol{r})$. In the following we consider $\rho_{k} \neq 0$ (local dependency of pixels in a given region), and $m(\boldsymbol{r})$ either a piecewise constant function $m_{k}(\boldsymbol{r})=m_{k}$, or a piecewise bilinear function of the position $\boldsymbol{r}$, i.e. $m_{k}(\boldsymbol{r})=m_{k}(x, y)=a_{k} \times x(\boldsymbol{r})+$ $b_{k} \times y(\boldsymbol{r})+m_{k}$. In the first case, each region $k$ is characterized by $\left(m_{k}, \sigma_{k}^{2}, \rho_{k}\right)$ and in the second case by $\left(a_{k}, b_{k}, m_{k}, \sigma_{k}^{2}, \rho_{k}\right)$. The priors on $\left(a_{k}, b_{k}\right)$ are chosen to be Gaussian laws with a mean equals to zero, and a large variance (so, it is almost equivalent to an uniform prior). From a posterior point of view, this is equivalent to use Gaussian laws with mean values estimated from the data and a low variance. FIGURE 1 illustrates the difference between constant (a) and bilinear model (c) for two images of size $200 \times 200$ pixels. (b) and (d) show pixel intensities on the $170^{\text {th }}$ row of both corresponding images. 


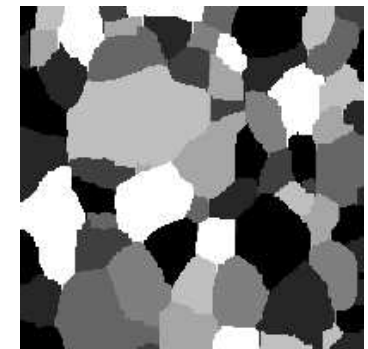

(a)

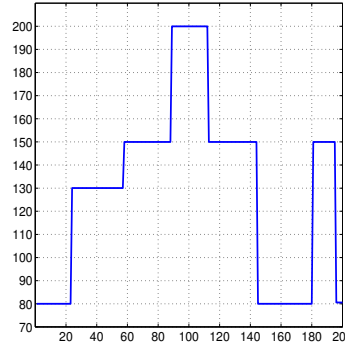

(b)

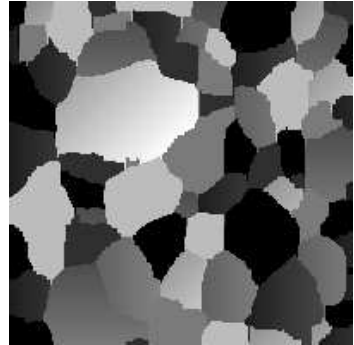

(c)

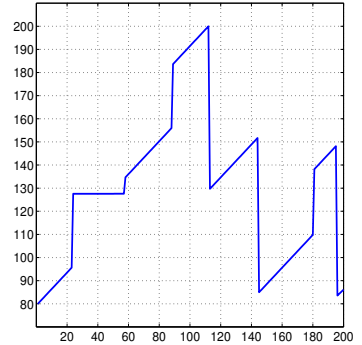

(d)

FIGURE 1. (a) image made of constant homogeneous regions, (b) $170^{\text {th }}$ row of (a), (c) image made of bilinear homogeneous regions, (d) $170^{\text {th }}$ row of (c).

\section{SIMULATION RESULTS}

\subsection{Synthetic Data with Bilinear Regions}

In the example presented in FIGURE 2, we began from the $200 \times 200$ pixels synthetic image made of homogeneous and bilinear regions (a) that we also degraded with a decimation factor equals to 4 and a $20 \mathrm{~dB}$ Gaussian noise using (1). We used the registration method presented in [2] to estimate the global shift between images. The real shift values are known because they are synthetical data, and in this noisy case, only $50 \%$ of the correct shifts are accurately estimated by the chosen registration method. One of the $8 \mathrm{LR}$ images of size $50 \times 50$ pixels obtained is shown on (d). Next, (b) and (e) are the HR image and its joint result of segmentation using the constant model, a SR factor of 4, and 8 labels for the segmentation's parametrization. (c) and (f) are the HR image and its joint segmentation using the same parameters but the bilinear model.

With this synthetic example made of bilinear regions and which corresponds well to our new model, we may notice that the HR image obtained with the bilinear method (c) is lightly better than the one obtained with the constant model (b). In particular, region's edges are sharper. Also, we can notice that reconstructed HR images are denoised in comparaison to LR image.

In comparison with $\widehat{\boldsymbol{f}}_{8 \text {, const. }}$, numerical criteria presented in TABLE 1 confirm that $\widehat{\boldsymbol{f}}_{8, \text { bil. }}$ is closest to the initial image $\boldsymbol{f}$. In this table, $\Delta_{\text {mean }}$ and $\Delta_{S D}$ stand for the mean and standard deviation of the difference image using $f$ as reference, and $\Delta_{1}$ is the L1 normalized relative error [1].

TABLE 1. Evaluation criteria of SR methods on synthetic data example.

\begin{tabular}{|c|c|c|c|c|c|}
\hline & $\begin{array}{l}\text { 2-D correlation } \\
\text { coefficient }\end{array}$ & $\begin{array}{c}\Delta_{\text {mean }} \\
\left(\times 10^{-2}\right)\end{array}$ & $\begin{array}{c}\Delta_{S D} \\
\left(\times 10^{-2}\right)\end{array}$ & $\begin{array}{c}\Delta_{1} \\
\left(\times 10^{-1}\right)\end{array}$ & $\begin{array}{c}\% \text { of error on } \\
\text { estimation of labels }\end{array}$ \\
\hline$\left(\widehat{\boldsymbol{f}}_{8, \text { const. }}, \widehat{\boldsymbol{z}}_{8, \text { const. }}\right)$ & 0.957 & 3.44 & 8.30 & 1.47 & 31.7 \\
\hline$\left(\widehat{\boldsymbol{f}}_{8, \text { bil. }}, \widehat{\boldsymbol{z}}_{8, \text { bil. }}\right)$ & 0.960 & 2.41 & 8.12 & 1.37 & 30.7 \\
\hline
\end{tabular}




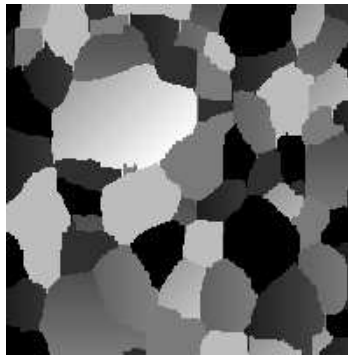

(a)

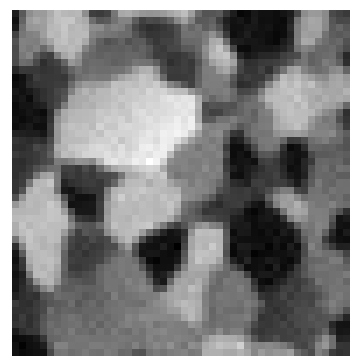

(d)

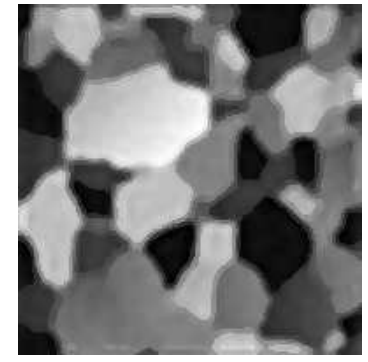

(b)

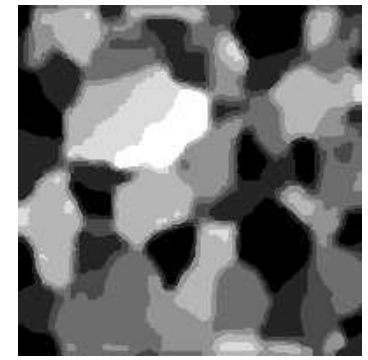

(e)

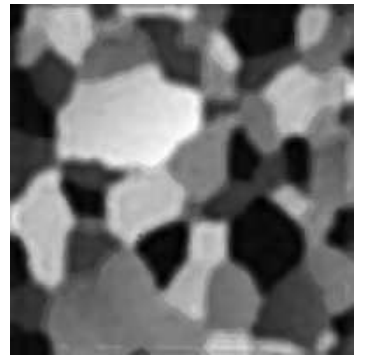

(c)

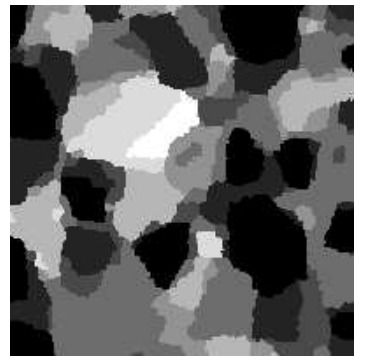

(f)

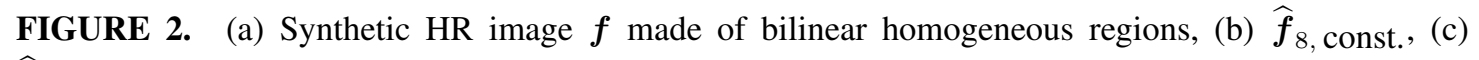
$\widehat{\boldsymbol{f}}_{8, \text { bil. }}$, (d) one of the 8 LR images used, (e) $\widehat{\boldsymbol{z}}_{8 \text {, const. }}$, (f) $\widehat{\boldsymbol{z}}_{8 \text {, bil. }}$

\subsection{Real Video Sequence}

FIGURE 3 shows the results obtained with our two methods applied to a real video sequence, with a SR factor of 4 and 8 labels for the segmentation's parametrization. One of the $70 \times 69$ pixels LR images used in these processes is shown on (c). Then, (a) and (d) are the HR image and its joint result of segmentation using the constant model, and (b) and (e) are the HR image and its joint segmentation using the bilinear model.

Here again, we can hardly notice any difference in both HR images obtained. However we might see that the "PHILIPS" tags on the batteries, which is unreadable on the LR image (c), is lightly more accurate on the HR image obtained with the bilinear method.

\section{CONCLUSIONS}

We presented in this communication an extension to a method of SR which is connected to a joint segmentation process and is realized in a Bayesian framework [1]. We have developed here a more complete model to define the region's homogeneity. We assume now that all pixel intensities in each given connexe homogeneous region follow a bilinear model.

We illustrated this new model with one synthetic example and one real video sequence. The obtained results show a light improvement in the reconstructed HR images using the bilinear model in comparison to the constant one. In the synthetic case, numerical criteria confirm the improvement brought by the bilinear model. In a more global perspective, we can see that HR images acquired with both methods show a lot more 

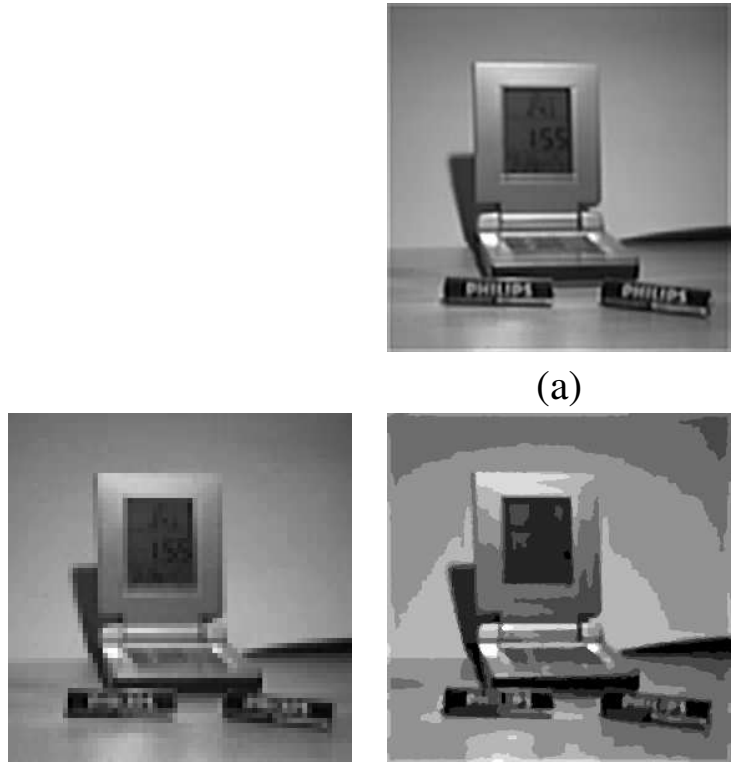

(c) (a)

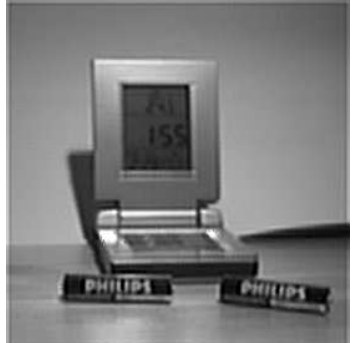

(b)

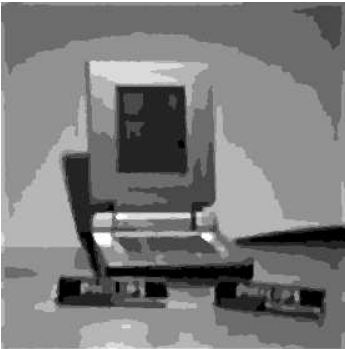

(d)

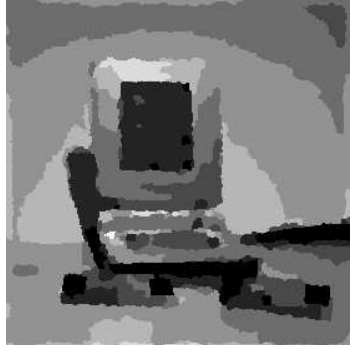

(e)

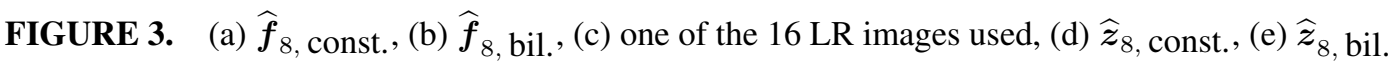

scene's details than the LR images, and they are correctly denoised. More, we obtain a segmentation's result which can be very useful to a posterior process such as geometrical feature extraction or detection.

\section{REFERENCES}

1. F. Humblot, and A. Mohammad-Djafari, "Super-Resolution using Hidden Markov Model and Bayesian Detection Estimation Framework," in EURASIP Journal on Applied Signal Proc., special issue on Super-Resolution Imaging Analysis, Algorithms, and Applications (to be published), 2005.

2. F. Humblot, B. Collin, and A. Mohammad-Djafari, "Evaluation and Practical Issues of Subpixel Image Registration Using Phase Correlation Methods," in PSIP Conference, Toulouse, France, 2005.

3. R. Tsai, and T. Huang, "Multi-Frame Image Restoration and Registration," in Advances in Computer Vision on Image Processing, 1984, vol. 1, pp. 317-339.

4. S. Borman, Topics in Multiframe Superresolution Restoration, Ph.D. thesis, University of Notre Dame, Notre Dame, IN (2004).

5. M. Hong, M. Kang, and A. Katsaggelos, "A Regularized Multichannel Restoration Approach for Globally Optimal High-Resolution Video Sequence," in SPIE Visual Communications and Image Processing, 1997, pp. 1306-1316.

6. N. Nguyen, P. Milanfar, and G. Golub, "A Computationally Efficient Image Superresolution Algorithm," in IEEE Trans. on Image Processing, 2001, vol. 10 (4), pp. 573-583.

7. S. Farsiu, D. Robinson, M. Elad, and P. Milanfar, "Fast and Robust Multi-Frame Super-Resolution," in IEEE Trans. on Image Processing, 2004, vol. 13 (10), pp. 1327-1344.

8. P. Cheeseman, B. Kanefsky, R. Kraft, J. Stutz, and R. Hanson, Super-Resolved Surface Reconstruction From Multiple Images, Tech. Rep. FIA-94-12, NASA Ames Research Center (1994).

9. R. Molina, J. Mateos, A. Katsaggelos, and M. Vega, "Bayesian Multichannel Image Restoration Using Compound Gauss-Markov Random Fields," in IEEE Trans. on Image Processing, 2003, vol. 12 (12), pp. 1642-1654.

10. H. Snoussi, Bayesian approach to source separation. Applications in imagery, Ph.D. thesis, University of Paris-Sud, Orsay, France (2003). 